\title{
miR-188-5p promotes oxaliplatin resistance by targeting RASA1 in colon cancer cells
}

\author{
XIJIA ZHU ${ }^{1}$, XISHUN LUO $^{1}$, ZHIKE SONG ${ }^{1}$, SHIYU JIANG $^{1}$, XIANGKAI LONG ${ }^{1}$, \\ XUEYUAN GAO ${ }^{2}$, XINYANG XIE ${ }^{2}$, LAIJIAN ZHENG ${ }^{2}$ and HAIPENG WANG ${ }^{1}$ \\ ${ }^{1}$ Department of Gastrointestinal Surgery, The Second Affiliated Hospital of Guilin Medical University, \\ Guilin, Guangxi Zhuang Autonomous Region 541199; ${ }^{2}$ Department of Gastrointestinal Surgery, \\ Guilin Medical University, Guilin, Guangxi Zhuang Autonomous Region 541004, P.R. China
}

Received September 30, 2020; Accepted February 5, 2021

DOI: $10.3892 / 01.2021 .12742$

\begin{abstract}
The efficacy of chemotherapy for colon cancer is limited due to the development of chemoresistance. MicroRNA (miR)-188-5p is downregulated in various types of cancer. The aim of the present study was to explore the molecular role of miR-188 in oxaliplatin (OXA) resistance. An OXA-resistant colon cancer cell line, SW480/OXA, was used to examine the effects of miR-188-5p on the sensitivity of colon cancer cells to OXA. The target of miR-188-5p was identified using a luciferase assay. Cell cycle distribution was also assessed using flow cytometry. The measurement of p21 protein expression, Hoechst 33342 staining and Annexin V/propidium iodide staining was used to evaluate apoptosis. The expression of miR-188-5p significantly increased in SW480/OXA compared with wild-type SW480 cells. The luciferase assay demonstrated that miR-188-5p inhibited Ras GTPase-activating protein 1 (RASA1; also known as p120/RasGAP) luciferase activity by binding to the 3 '-untranslated region of RASA1 mRNA, suggesting that miR-188-5p could target RASA1. In addition, miR-188-5p downregulation or RASA1 overexpression promoted the chemosensitivity of SW480/OXA, as evidenced by increased apoptosis and $\mathrm{G}_{1} / \mathrm{S}$ cell cycle arrest. Moreover, RASA1 silencing abrogated the increase in cell apoptosis induced by the miR-188-5p inhibitor. The findings of the present study suggested that miR-188-5p could enhance colon cancer cell chemosensitivity by promoting the expression of RASA1.
\end{abstract}

Correspondence to: Professor Haipeng Wang, Department of Gastrointestinal Surgery, The Second Affiliated Hospital of Guilin Medical University, 212 Renmin Road, Lingui, Guilin, Guangxi Zhuang Autonomous Region 541199, P.R. China

E-mail: wanghaipeng201605@163.com; 215833387@qq.com

Abbreviations: CRC, colorectal cancer; miR/miRNA, microRNA; OXA, oxaliplatin; RASA1, Ras GTPase-activating protein 1; UTR, untranslated region

Key words: chemoresistance, miR-188-5p, colon cancer, oxaliplatin, RASA1, apoptosis

\section{Introduction}

Colon cancer is the third most frequently diagnosed cancer and the second leading cause of cancer death in the United States (1). In the United States, 104,610 new cases of colon cancer were reported in 2020 (1). Among them, approximately 53,200 mortalities were reported annually in the United States (1). Overall, the incidence and death rates for colon cancer are on the decline (2). However, this trend is not observed in younger individuals from 15-35 years old (1-3). The two major causes of colon cancer are chromosomal instability and serrated neoplasia (3). Treatment options for colon cancer include endoscopic and surgical local excision, preoperative radiotherapy and chemotherapy (3). However, chemoresistance remains an obstacle to the treatment of colon cancer $(4,5)$.

MicroRNA (miRNA/miR) plays a significant role in complex physiological and pathological processes through the regulation of target gene expression via binding to their 3'-untranslated region (UTR) (6). Usually, each miRNA has multiple target mRNA transcripts, resulting in various effects, such as cell proliferation, apoptosis, migration and invasion (7). Previous studies have highlighted the link between miRNA and the chemoresistant phenotype of different tumor types, which is mediated by abnormal regulation of apoptosis (8), cell cycle distribution (9) and activity of drug efflux transporters (10). Modulating the expression of miRNA using specific mimics or inhibitors normalizes gene regulatory networks and signaling pathways, thus sensitizing cancerous cells to chemotherapy (11). Therefore, miRNA-based gene therapy may represent an attractive approach for cancer therapy $(11,12)$.

In various types of cancer, such as cervical cancer (13), non-small cell lung cancer (14), gastric cancer (15), glioma (16), colorectal cancer (CRC) (17), oral squamous cell carcinoma (18), hepatocellular carcinoma (19), prostate cancer (20), acute myeloid leukemia (21) and rectal cancer (22), miR-188-5p is downregulated. Moreover, transfection of miR-188-5p mimics reduces proliferation and migration while promoting apoptosis in A549 and H2126 cells (14). In in vivo xenograft models, miR-188-5p inhibits tumor growth of non-small cell lung cancer (14). Additionally, miR-188-3p regulates the expression of myeloid/lymphoid or mixed-lineage leukemia 4 (MLLT4) and promotes the migration of HCT116 and HRT18 
cancer cells (17); it has also been proposed as a novel independent prognostic factor in patients with CRC (17). In locally advanced rectal cancer, miR-188-5p is associated with a complete pathological response to the neoadjuvant chemoradiotherapy (22). However, the role of miR-188 in drug-resistant cancer cells has yet to be examined.

Ras GTPase-activating protein 1 (RASA1; also known as p120/RasGAP) was the first identified RasGAP protein. RASA1 has been implicated in a number of biological processes, including actin filament polymerization, cell apoptosis and migration (23). RASA1 acts as a cancer suppressor gene in several types of tumor (24-32). RASA1 suppresses the effect of Ras proteins by enhancing their weak intrinsic GTPase activity, leading to an increase in the levels of the inactive, GDP-bound form of Ras (33). This causes aberrant intracellular signaling through the Ras-RAF-ERK pathway (33). A previous study has demonstrated that RASA1 protein levels are significantly decreased in colon cancer cells and that RASA1 is a target of miR-21, which promotes the malignancy of colon cancer cells (34). The upregulation of RASA1 inhibits cell proliferation and inhibits the RAS signaling pathway (34). Moreover, the expression levels of miR-223 and RASA1 are inversely correlated in tumor tissue from patients with CRC (35). RASA1 is also a validated target of miR-335, which is downregulated in CRC (36). Several studies have also indicated that onco-miR molecules, such as miR-21 and miR-182, promote tumor angiogenesis and lymph node metastasis by targeting RASA1 $(37,38)$.

The present study aimed to explore the molecular role of miR-188-5p in oxaliplatin (OXA) resistance. miR-188-5p may enhance colon cancer cell chemosensitivity by promoting the expression of RASA1, providing a new insight into treatment options for OXA-resistant colon cancer.

\section{Materials and methods}

Cell culture and treatment. The human SW480 colon cancer cell line was purchased from American Type Culture Collection. To induce OXA resistance in SW480 cells (SW480/OXA), SW480 cells were seeded into a 24-well plate at a density of $1 \times 10^{5}$ cells/well and cultured in the presence of 0.5 mM OXA (Sigma-Aldrich; Merck KGaA; cat. no. O9512) for $24 \mathrm{~h}$. Subsequently, the medium was replaced with OXA-free medium, and the cells were cultured for three days. From day 4, the drug concentration increase started. This procedure was continued for 6 months with a drug concentration increase at $0.4 \mu \mathrm{M} /$ month, $(0.5-2.5 \mathrm{mM})$ and the medium was changed at every other day. After appropriately increasing the concentration of OXA over a period of 6 months, a stable drug-resistant cell line SW480/OXA was obtained. Cells were cultured in Dulbecco's modified Eagle's medium (DMEM; Sigma-Aldrich; Merck KGaA) with 10\% Fetal Bovine Serum (FBS; Gibco; Thermo Fisher Scientific Inc.) and 1\% penicillin/streptomycin solution at $37^{\circ} \mathrm{C}$ with $5 \% \mathrm{CO}_{2}$ saturated humidity.

Hoechst 33342 staining. SW480 and SW480/OXA cells ( $2 \times 10^{5} /$ well) were seeded into a fibronectin-coated 12 -well plate. After cell transfection, $5 \mu \mathrm{g} / \mathrm{ml}$ Hoechst 33342 staining solution (Beijing Solarbio Science \& Technology Co., Ltd.; cat. no. C0031) was added to the cells, which were incubated for $20 \mathrm{~min}$ at room temperature. The cells were then washed three times with PBS. Coverslips were sealed and visualized under a confocal microscope (T1-SAM microscope; Nikon Corporation).

Reverse transcription-quantitative (RT- $q$ ) PCR. Total RNA was isolated from SW480 and SW480/OXA cells $\left(5 \times 10^{5}\right.$ cells/well in a 6 -well plate) using TRIzol ${ }^{\circledR}$ (Invitrogen; Thermo Fisher Scientific, Inc.; cat. no. 15596026) according to the manufacturer's instructions. First-strand cDNA was synthesized using the High-Capacity cDNA Reverse Transcription kit (Applied Biosystems; Thermo Fisher Scientific, Inc.; cat. no. 4368814) according to the manufacturer's protocol. SYBR Green PCR Master mix (Applied Biosystems; Thermo Fisher Scientific, Inc.; cat. no. A25741) was used to perform RT-qPCR. The qPCR thermocycling conditions were as follows: Initial denaturation at $94^{\circ} \mathrm{C}$ for $5 \mathrm{~min}$, followed by 40 cycles of denaturation at $94^{\circ} \mathrm{C}$ for $15 \mathrm{sec}$, annealing at $60^{\circ} \mathrm{C}$ for $25 \mathrm{sec}$ and extension at $72^{\circ} \mathrm{C}$ for $30 \mathrm{sec}$. U6 and GAPDH were used as internal references for normalization. The $2^{-\Delta \Delta \mathrm{Cq}}$ method was applied to calculate relative mRNA levels (36). The primer sequences used in the study were: i) GAPDH forward, 5'-TGTTCGTCATGGGTGTGAAC-3' and reverse, 5'-ATGGCATGGACTGTGGTCAT-3'; ii) RASA1 forward, 5'-CAGTGGACGAAGGTGACTCT-3' and reverse, 5'-AGG CGTTCTTCTGCTATCGT-3'; iii) U6 forward, 5'-CTCGCT TCGGCAGCACA-3' and reverse, 5'-AACGCTTCACGA ATTTGCGT-3'; and iv) miR-188-5p forward, 5'-CTCAAC TGGTGTCGTGGAGT CGGCAATTCAGTTGAGCCCTC CAC-3' and reverse, 5'-ACACTCCAGCTGGGCATCCCT TGCATGGTGG-3'.

Cell transfection. The miR-188-5p inhibitor (5'-CCCUCCACC AUGCAAGGGAUG-3'; 2'-OMe-modified), negative control (NC) NC inhibitor (5'-CAGUACUUUUGUGUAGUACAA-3'), miR-188-5p mimics (sense, 5'-CAUCCCUUGCAUGGUGGA GGG-3'; antisense, 5'-CUCCACCAUGCAAGGGAUGUU-3'), NC mimics (sense, 5'-UUCUCCGAACGUGUCACGUTT-3'; antisense, 5'-ACGUGACACGUUCGGAGAATT-3'). The mimic and inhibitor NCs were scrambled sequences. RASA1 small interfering (si)RNA (5'-CAGTTTATGATGGGAGGCC GGTATT-3'), siRNA negative control (siNC, 5'-TTCTCC GAACGTTTCACGTTT-3'), RASA1 overexpression plasmid (pcDNA3.1-RASA1) and empty vector control (pcDNA3.1) were purchased from Shanghai GenePharma Co. Ltd. Cells were seeded in a 12 -well plate $\left(4 \times 10^{4}\right.$ cells/well) and incubated overnight at $37^{\circ} \mathrm{C}$ with $5 \% \mathrm{CO}_{2}$. At $70 \%$ confluency, cells were transfected with 100 pmol miR-188-5p mimics or NC mimics at room temperature for $48 \mathrm{~h}$ using Lipofectamine ${ }^{\circledR} 2000$ (cat. no. 11668-500; Invitrogen; Thermo Fisher Scientific, Inc.) according to the manufacturer's instructions. SW480 and SW480/OXA cells were transfected with 50 pmol si-RASA1 or co-transfected with $50 \mathrm{pmol}$ miR-188-5p inhibitor and si-RASA1. After $48 \mathrm{~h}$, cells were collected for subsequent experiments.

Bioinformatics analysis. For the determination of potential target genes of miR-188-5p, various bioinformatics tools, including TargetScan (http://www.targetscan.org), miRDB 
(http://www.mirdb.org/) and PITA (http://genie.weizmann. ac.il/pubs/mir07/mir07_data.html) databases were used. TargetScan was used to predict the binding mRNAs (218 mRNAs), similarly, 340 mRNAs and 229 mRNAs were predicted in miRDB and PITA databases, respectively. When the common mRNAs from the three databases were assessed, 6 mRNAs were obtained. RASA1 was one of the predicted target genes of miR-188-5p.

Luciferase activity assay. Luciferase reporter constructs were obtained by ligating the wild-type (WT) or mutant (MUT) version of the RASA1 3'-untranslated region (UTR) to the psiECHECK-2 vector containing a luciferase reporter gene (Promega Corporation; cat. no. C8021). SW480 and SW480/OXA cells $\left(5 \times 10^{5} /\right.$ well $)$ were seeded into 6 -well plates and co-transfected with RASA1 WT or MUT and with miR-188-5p mimics or NC mimics using Lipofectamine $2000^{\circledR}$ (Invitrogen; Thermo Fisher Scientific, Inc.; cat. no. 11668-500). A Luciferase Reporter Gene Detection kit (Sigma-Aldrich; Merck KGaA; LUC1-1KT) was used to detect luciferase activity $48 \mathrm{~h}$ after transfection. Luciferase activity was normalized to the Renilla luciferase activity. Experiments were set up in triplicate and performed independently three times.

Western blot analysis. SW480 and SW480/OXA cells $\left(1 \times 10^{6}\right.$ cells $/ 6-\mathrm{cm}$ dish) were collected and lysed in lysis buffer containing phosphorylase inhibitor cocktail (Roche; Diagnostics; cat. no. 04693124001) and phenylmethanesulfonyl fluoride. Protein concentration was measured using the bicinchoninic acid (BCA) Protein Concentration Determination kit (Beyotime Institute of Biotechnology) following the manufacturer's instructions. An equal amount $(30 \mu \mathrm{g})$ of each protein sample was resolved by SDS-PAGE on $8-12 \%$ gels, then transferred to PVDF membranes. Subsequently, the membranes were blocked using $5 \%$ skimmed milk powder for $1 \mathrm{~h}$ at room temperature, then incubated with primary antibodies at $4^{\circ} \mathrm{C}$ overnight. The primary antibodies were specific for p21 (Abcam; cat. no. ab227443; 1:1,000), RASA1 (Abcam; cat. no. ab2922; 1:1,000), Rs-GTP (Abcam; cat. no. ab69747; 1:2,000) and GAPDH (Abcam; cat. no. ab9485; 1:5,000). Goat anti-rabbit (Sigma-Aldrich; Merck KGaA; cat. no. 1:5,000) or goat anti-mouse IgG-HRP (Sigma-Aldrich; Merck KGaA; cat. no. AP-308P; 1:5,000) secondary antibodies were then added at room temperature for $1 \mathrm{~h}$. The expression of the target proteins was normalized to the GAPDH internal control. The protein bands were visualized using an enhanced chemiluminescence kit (Beyotime Institute of Biotechnology) according to the manufacturer's instructions and quantified using the Gel-Pro-Analyzer v.4.0 software (Media Cybernetics, Inc.)

Immunofluorescence assay. SW480 and SW480/OXA cells ( $2 \times 10^{5} /$ well) were seeded on a fibronectin-coated 12 -well plate. After transfection with indicated mimics or inhibitors, the cells were fixed in $4 \%$ paraformaldehyde at room temperature for 10 min, then blocked with $1 \%$ BSA in PBS. The slides were incubated with a RASA1-specific primary antibody (1:1,000; Abcam; cat. no. ab2922) at room temperature for $1 \mathrm{~h}$, then washed three times with PBS. An Alexa Fluor 555-conjugated goat anti-mouse secondary antibody (1:2,000; Abcam; cat. no. ab150114) was then added at room temperature for $40 \mathrm{~min}$. The slides were washed with PBS three times and then stained with DAPI at room temperature for $10 \mathrm{~min}$. For fluorescence visualisation, the Nikon $\mathrm{C} 1$ confocal microscope was used (Nikon Corporation; magnification, x100).

Apoptosis assay. SW480 and SW480/OXA cells (2x10\%/well) were seeded onto a 6-well plate and transfected with siRNA or plasmid as indicated. After $48 \mathrm{~h}$, the Annexin V-FITC apoptosis detection kit (BD Biosciences; cat. no. 556547) was used to detect apoptotic cells with Annexin V-FITC and propidium iodide (PI) double staining according to the manufacturer's instructions. Gated Annexin $\mathrm{V}^{+} / \mathrm{PI}^{+}$cells as late stage apoptosis and Annexin $\mathrm{V}^{+} / \mathrm{PI}^{-}$cells as early stage apoptosis were assessed. The analysis was performed using a BD FACScan ${ }^{\circledR}$ II flow cytometer (BD Biosciences), and the data were analyzed using CellQuest Pro software version 5.1 (Becton, Dickinson and Company).

Cell cycle analysis. Cells ( $2 \times 10^{5}$ cells/well) were seeded onto a 6 -well plate and transfected $12 \mathrm{~h}$ later. The cells were harvested $48 \mathrm{~h}$ after transfection, fixed in ice-cold $70 \%$ ethanol for at $4^{\circ} \mathrm{C}$ for $12 \mathrm{~h}$, washed with PBS, then stained with $5 \mathrm{mg} / \mathrm{ml}$ propidium iodide (Sigma-Aldrich; Merck KGaA, cat. no. P4170) or isotype control antibody IgG2A (10 $\mu 1 / 10^{6}$ cells; R\&D Systems; cat. no. IC003A) in PBS supplemented with RNase A (Roche Diagnostics; cat. no. 10154105103) for $30 \mathrm{~min}$ at room temperature. The flow cytometry data were acquired using a FACSCalibur ${ }^{\circledR}$ (BD Biosciences) flow cytometer. Flow cytometry data were analyzed using Summit v.5.3 (DAT/EM Systems International). A histogram was plotted according to the distribution of nuclear DNA content and the frequency of cells in each phase of the cell cycle was determined based on their DNA ploidy profile.

Statistical analysis. The data are presented as the mean \pm SD and were analyzed using SPSS 15.0 (SPSS, Inc.) All results are representative of at least three independent experiments unless stated otherwise. Unpaired two-tailed Student's t-test was used for comparisons between two groups. For multi-group comparisons, one-way ANOVA followed by Tukey's post hoc test was used. $\mathrm{P}<0.05$ was considered to indicate a statistically significant difference.

\section{Results}

Inhibition of miR-188-5p upregulates RASA1 expression. RT-qPCR was performed using SW480 and SW480/OXA cells to determine the expression levels of miR-188-5p. As shown in Fig. 1A, the expression of miR-188-5p was significantly upregulated in SW480/OXA cells compared with that in SW480 cells $(\mathrm{P}<0.001)$, suggesting that the expression of miR-188-5p may be associated with drug resistance in colon cancer cells. Bioinformatics analysis predicted that miR-188-5p potentially bound to RASA1 mRNA. There was a targeted binding association between miR-188-5p and RASA1. Moreover, mRNA expression levels of RASA1 were decreased in SW480/OXA cells compared with those in SW480 cells (Fig. 1B; P<0.001). To confirm whether RASA1 was a direct target of miR-188-5p, a luciferase assay was performed. Co-transfection with 

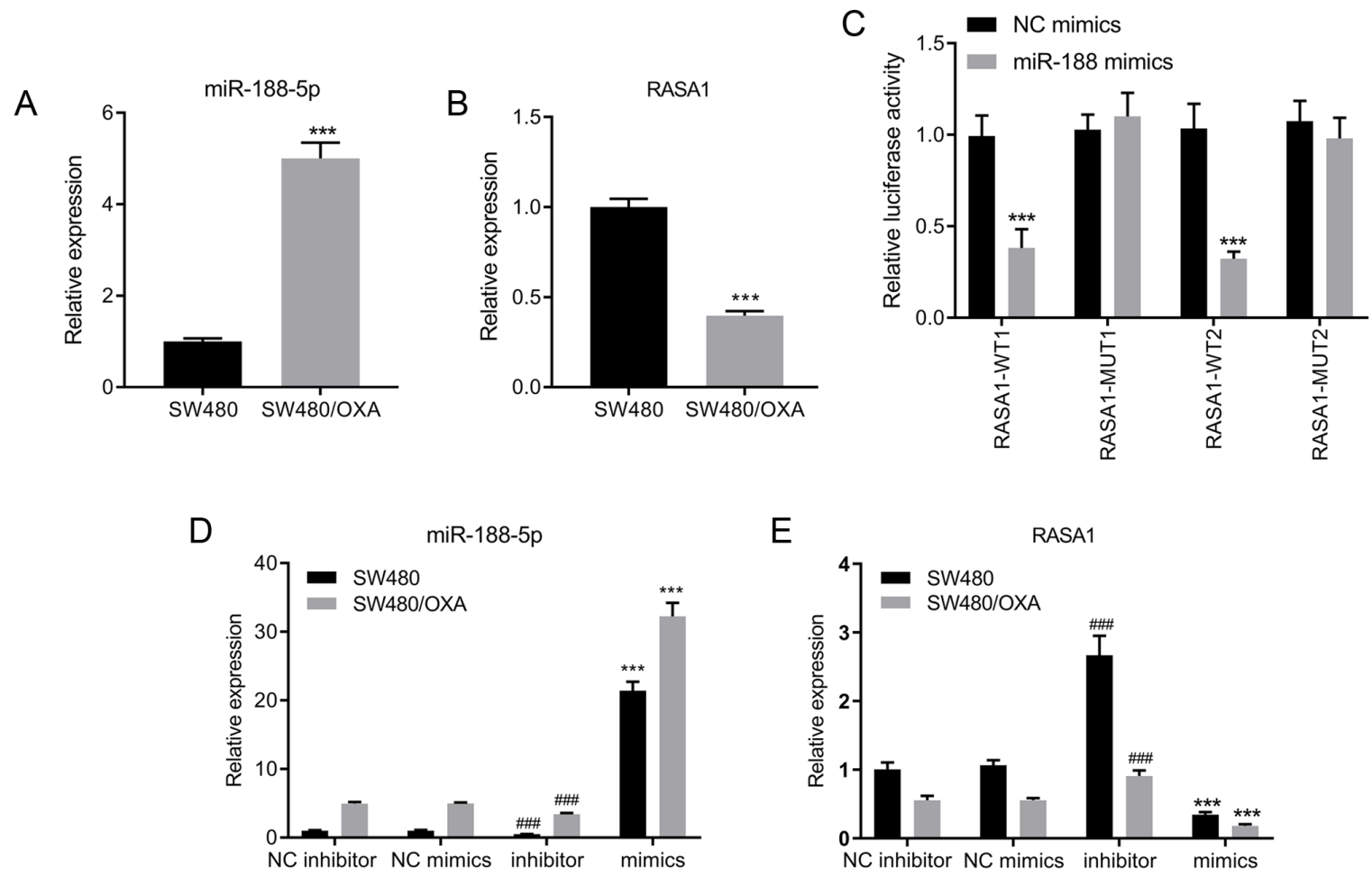

$\mathrm{F}$
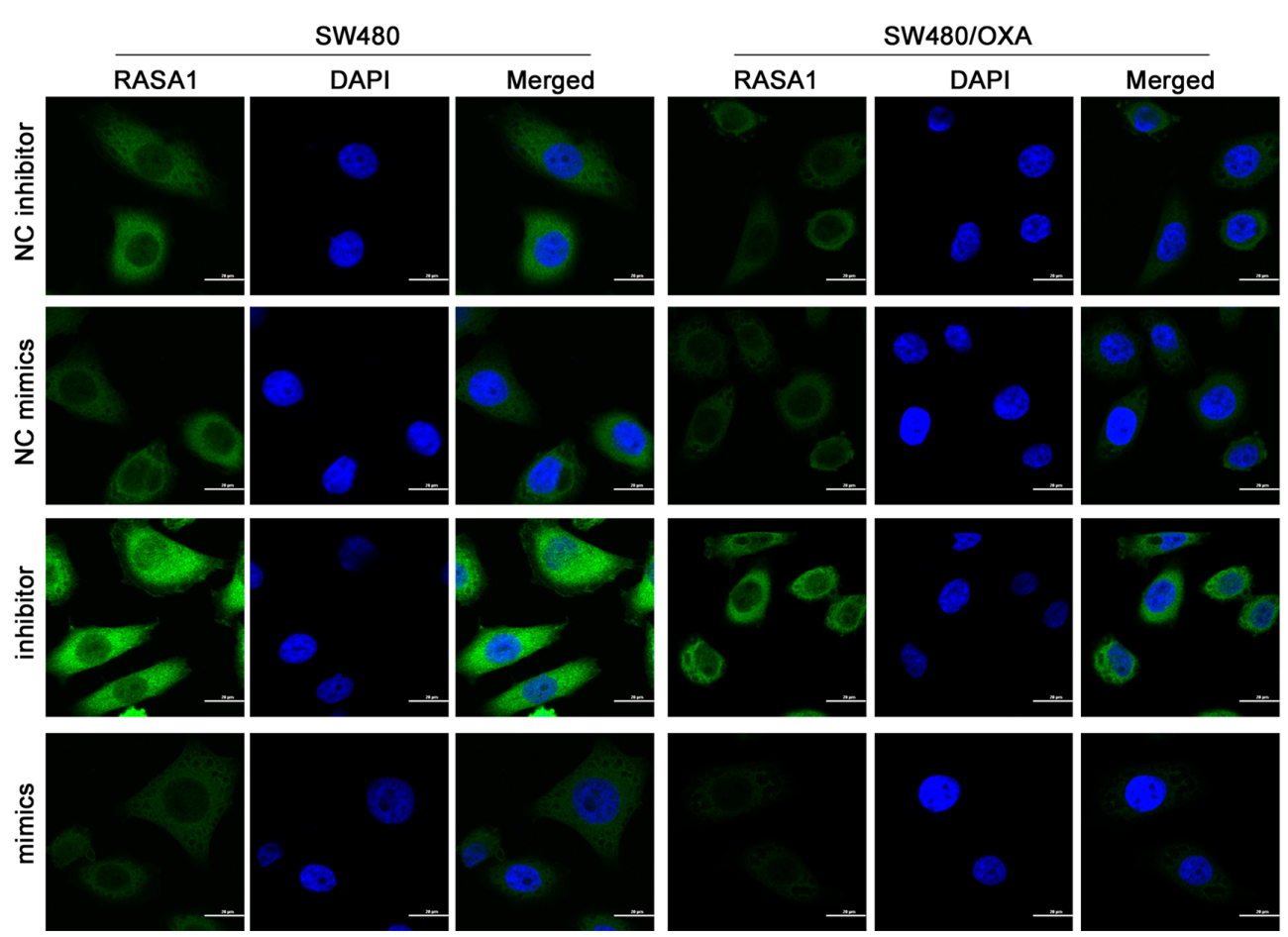

Figure 1. Downregulation of miR-188-5p upregulates RASA1 expression in colon cancer. (A and B) mRNA expression of (A) miR-188-5p and (B) and RASA1 in SW480 or SW480/OXA cells. ${ }^{* * *} \mathrm{P}<0.001$ vs. SW480. (C) Luciferase activity in SW480 cells co-transfected with indicated plasmids and miR-188-5p or NC mimics. ${ }^{* * *} \mathrm{P}<0.001$ vs. NC mimics. (D and E) mRNA expression of (D) miR-188-5p and (E) RASA1 in SW480 or SW480/OXA cells transfected with miR-188-5p mimics or inhibitor. ${ }^{* * * *} \mathrm{P}<0.001$ vs. NC mimics; ${ }^{\# \# \#} \mathrm{P}<0.001$ vs. NC inhibitor. (F) RASA1 expression in SW480 or SW480/OXA cells transfected with miR-188-5p mimics or inhibitor. Scale bar, $20 \mu \mathrm{m}$. miR, microRNA; oxa, oxaliplatin; RASA1, Ras GTPase-activating protein 1; WT, wild-type; MUT, mutant; NC, negative control.

miR-188-5p mimics significantly reduced the luciferase activity of WT RASA1, but not MUT RASA1, compared with NC mimics (Fig. 1C; $\mathrm{P}<0.001$ ). These results indicated that RASA1 was a target of miR-188-5p.
SW480 or SW480/OXA cells were transfected with miR-188-5p mimics or inhibitor to examine the regulatory effects of miR-188-5p on RASA1 expression. Transfection with miR-188-5p mimics significantly increased miR-188-5p 
A

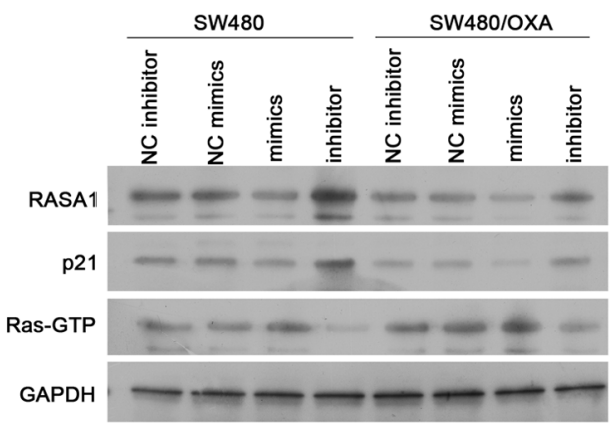

B

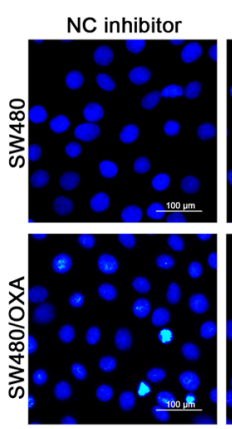

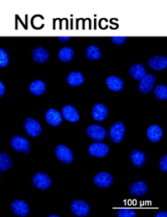
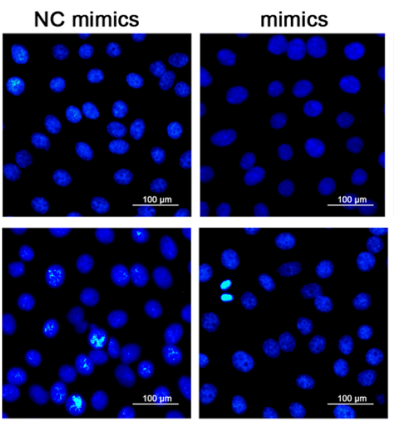

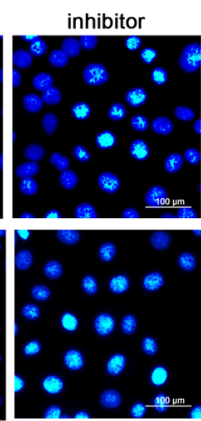

C
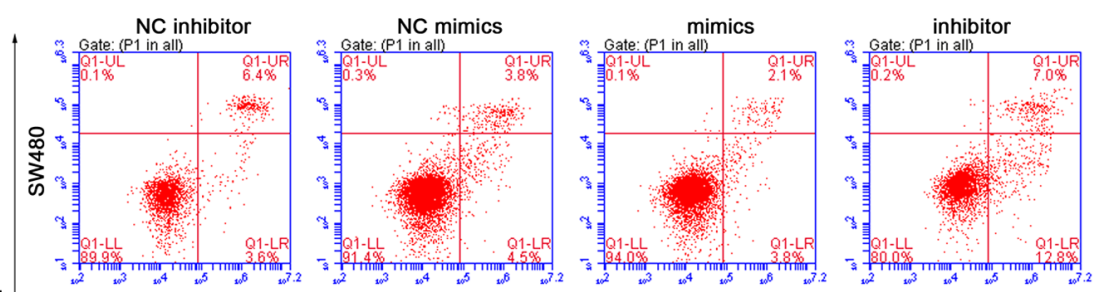

$\bar{a}$
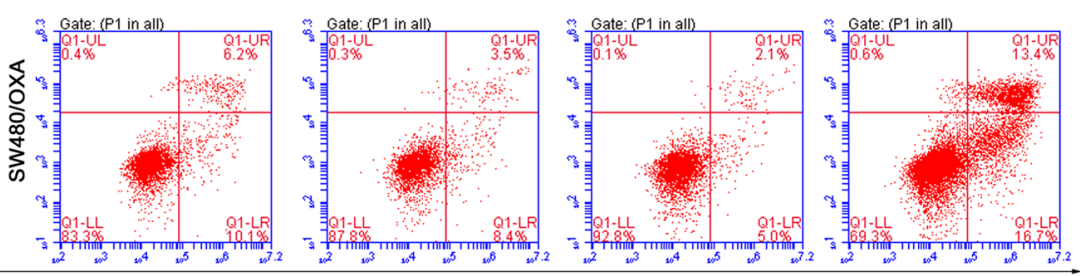

Annexin V-FITC

D

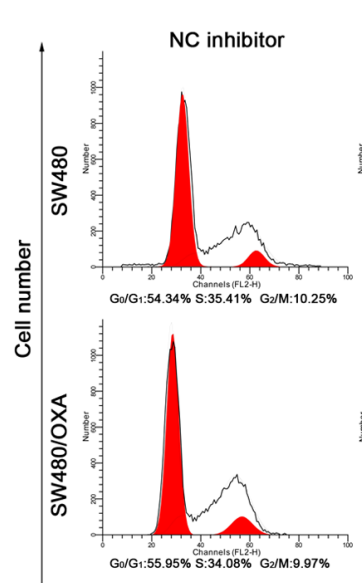

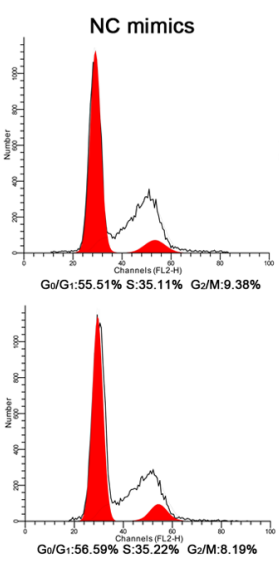
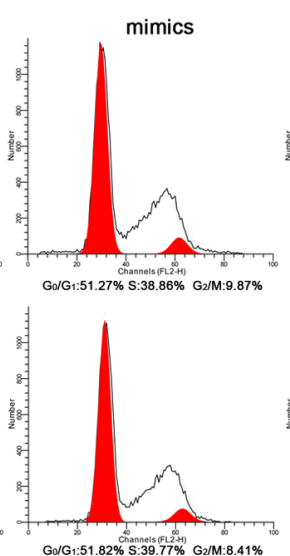

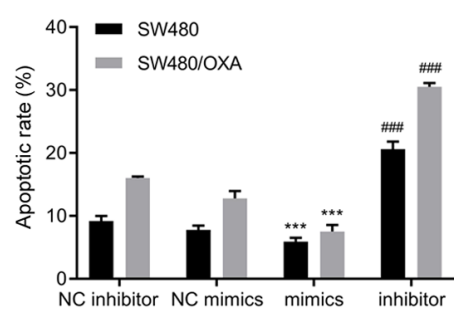

SW480

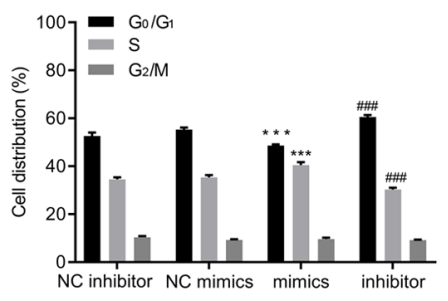

SW480/OXA
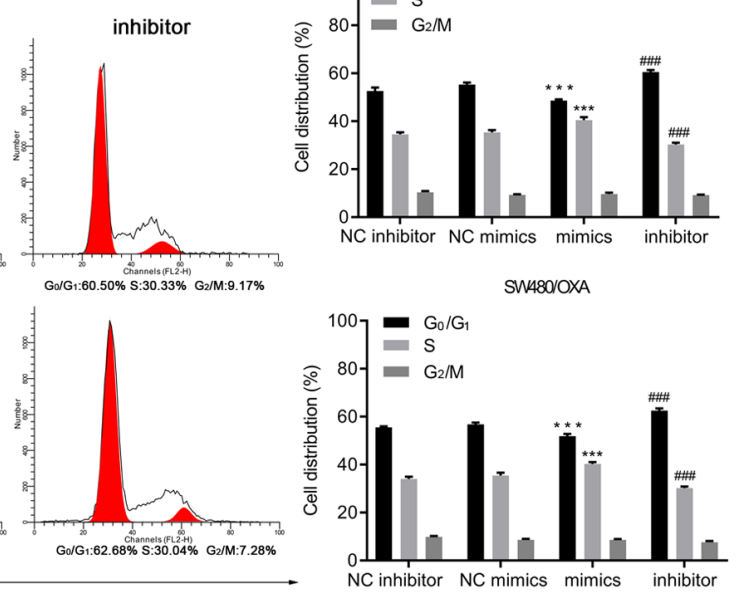

Figure 2. Downregulation of miR-188-5p induces cell apoptosis in SW480/OXA cells. (A) Protein expression levels of RASA1, p21, and Ras-GTP in SW480 or SW480/OXA cells transfected with miR-188-5p mimics or inhibitors. (B) Hoechst 33342 staining of SW480 or SW480/OXA cells transfected with miR-188-5p mimics or inhibitor. Scale bar, $100 \mu \mathrm{m}$. (C) Apoptosis of SW480 or SW480/OXA cells transfected with miR-188-5p mimics or inhibitor. ${ }^{* * *} \mathrm{P}<0.001$ vs. NC mimics; ${ }^{\# \# "} \mathrm{P}<0.001$ vs. NC inhibitor. (D) Cell cycle distribution was analyzed by flow cytometry. ${ }^{* * *} \mathrm{P}<0.001$ vs. NC mimics, ${ }^{\# \# \#} \mathrm{P}<0.001$ vs. NC inhibitor. miR, microRNA; oxa, oxaliplatin; RASA1, Ras GTPase-activating protein 1; PI, propidium iodide; FITC, fluorescein isothiocyanate; NC, negative control.

expression and reduced RASA1 expression, compared with the respective negative controls. By contrast, miR-188-5p inhibitor transfection reduced miR-188-5p expression and increased RASA1 expression(Fig. 1D and E; all $\mathrm{P}<0.001$ ). Moreover, immunofluorescence was used to detect the expression of RASA1, and the results suggested that transfection with miR-188-5p mimics decreased the expression of RASA1 at the protein level (Fig. 1F). Thus, inhibition of miR-188-5p increased the expression of its target gene, RASA1, both in SW480 and in SW480/OXA cells.
Targeting miR-188-5p promotes apoptosis in SW480/OXA cells. Transfections with miR-188-5p mimics or inhibitor were performed in SW480 or SW480/OXA cells to further examine the role of miR-188-5p on the apoptosis of colon cancer cells. The miR-188-5p mimics significantly decreased the protein expression levels of RASA1, compared with NC mimics; whereas miR-188-5p inhibitor significantly increased the expression of RASA1, compared with NC inhibitor $(\mathrm{P}<0.001$; Figs. 2A and S1A). In addition, the expression of p21 decreased 
A

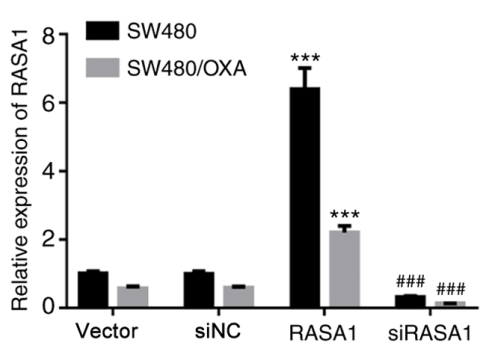

B

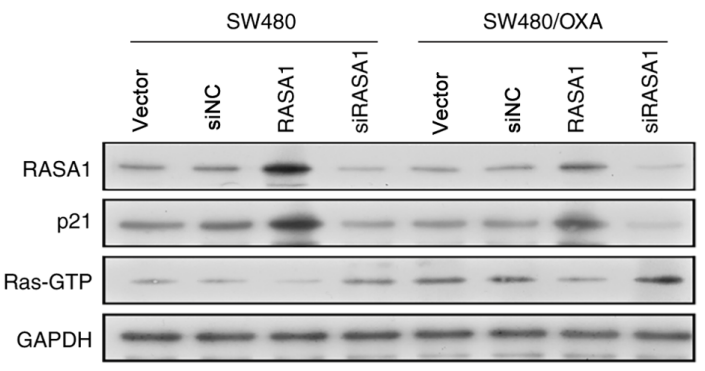

C
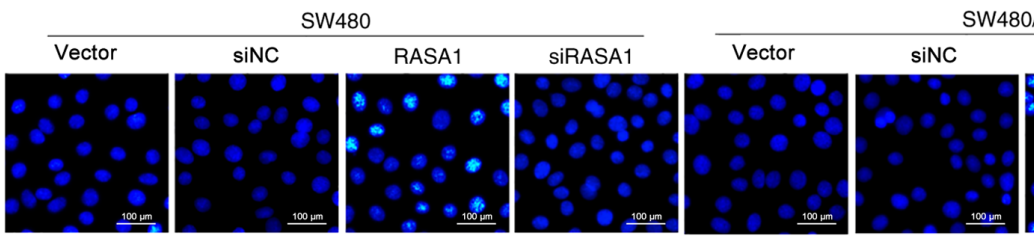

SW480/OXA

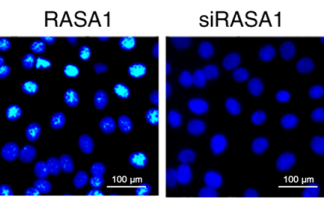

D
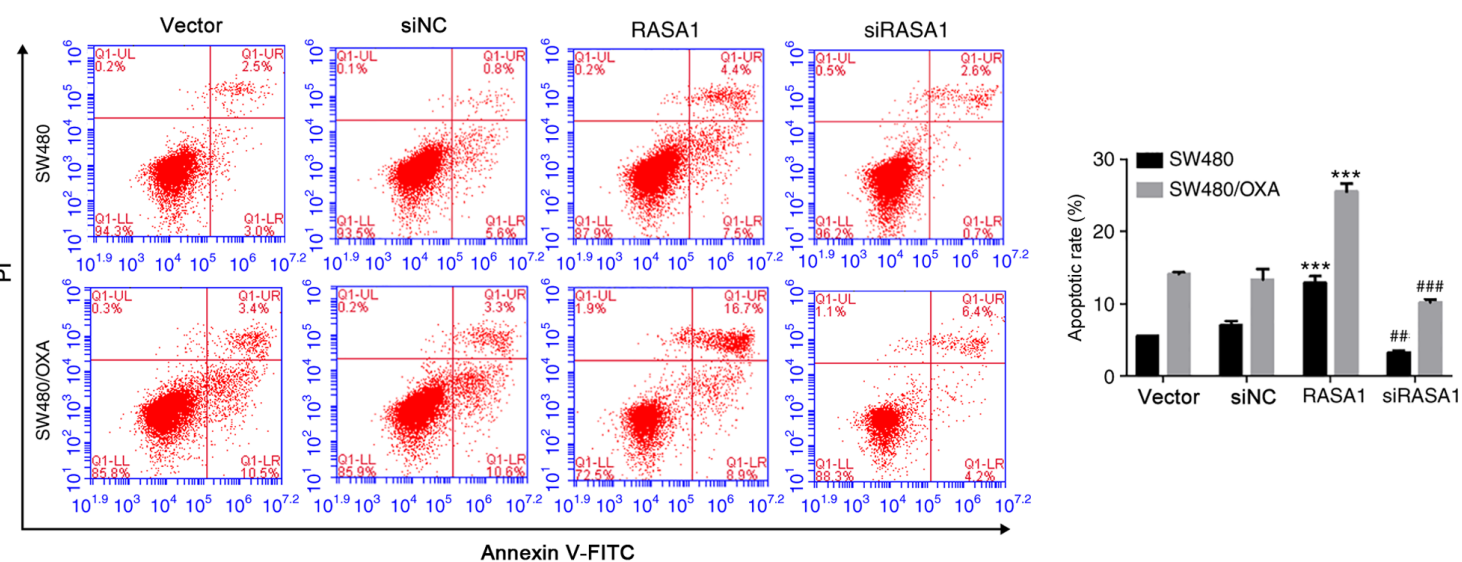

E
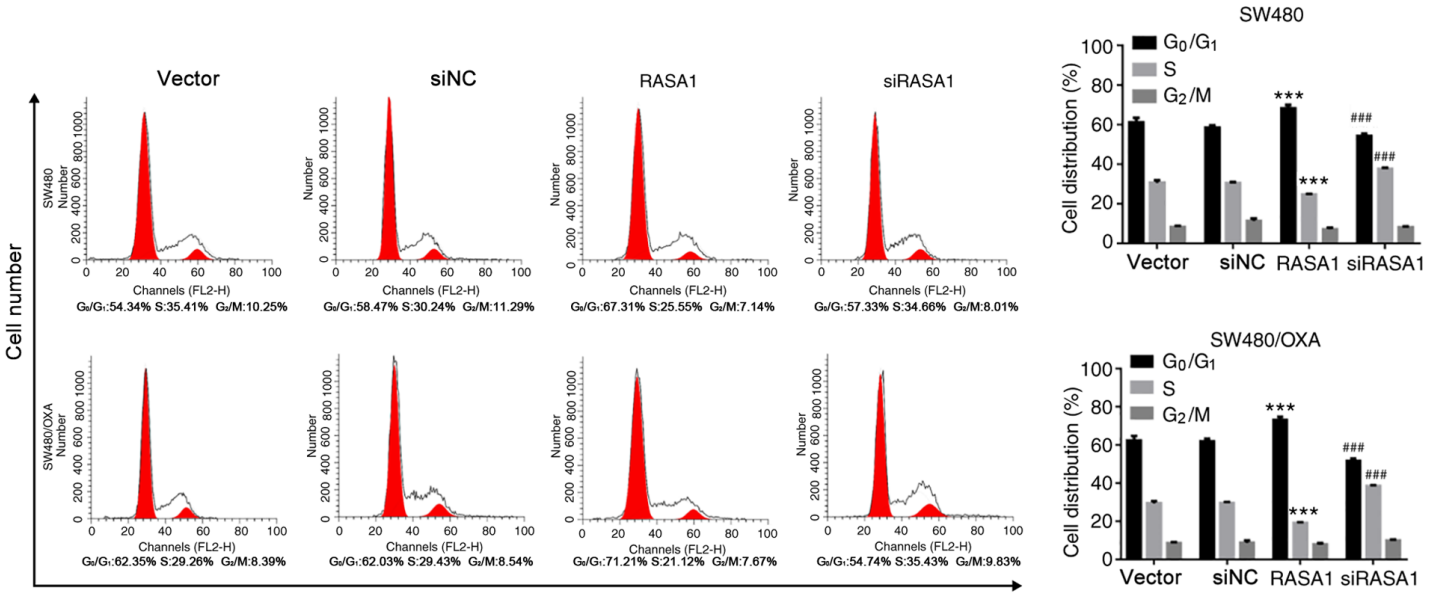

Figure 3. Overexpression of RASA1 accelerates apoptosis and inhibits the cell cycle. (A) Relative mRNA expression of RASA1 in SW480 or SW480/OXA cells transfected with RASA1 overexpression plasmid or RASA1 siRNA. ${ }^{* * *} \mathrm{P}<0.001$ vs. vector, ${ }^{\# \# \# ~} \mathrm{P}<0.001$ vs. siNC group. (B) RASA1, p21, and Ras-GTP protein expression in SW480 or SW480/OXA cells transfected with RASA1 plasmid or RASA1 siRNA. (C) Hoechst 33342 staining in SW480 or SW480/OXA cells transfected with RASA1 plasmid or RASA1 siRNA. Scale bar, $100 \mu \mathrm{m}$. (D) Apoptosis rate in SW480 or SW480/OXA cells following transfection with RASA1 plasmid or RASA1 siRNA. ${ }^{* * *} \mathrm{P}<0.001$ vs. vector, ${ }^{\# \# "} \mathrm{P}<0.001$ vs. siNC group. (E) Cell cycle distribution was analyzed by flow cytometry. ${ }^{* * *} \mathrm{P}<0.001$ vs,

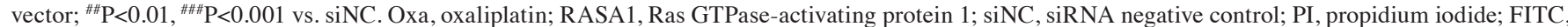
fluorescein isothiocyanate.

in miR-188-5p mimic-transfected cells and increased in miR-188-5p inhibitor-transfected cells, compared with that in $\mathrm{NC}$ mimics and $\mathrm{NC}$ inhibitor, respectively (Fig. 2A). Moreover, the expression of p21 was lower in miR-188-5p mimic-transfected SW480/OXA cells compared with that in mimic-transfected SW480 cells (Figs. 2A and S2A). As RASA1 suppresses the function of Ras and enhances the weak intrinsic GTPase activity of Ras proteins (34), the protein expression levels of Ras-GTP were then examined. The results demonstrated that miR-188-5p mimics significantly promoted 
A

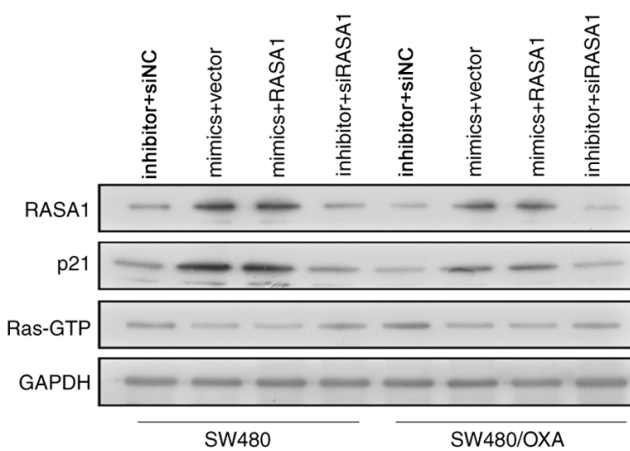

B

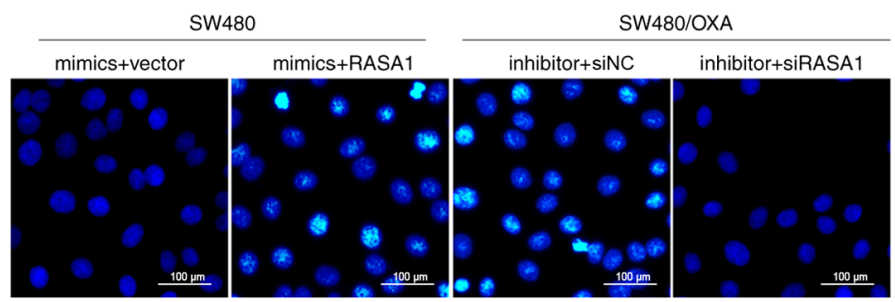

C
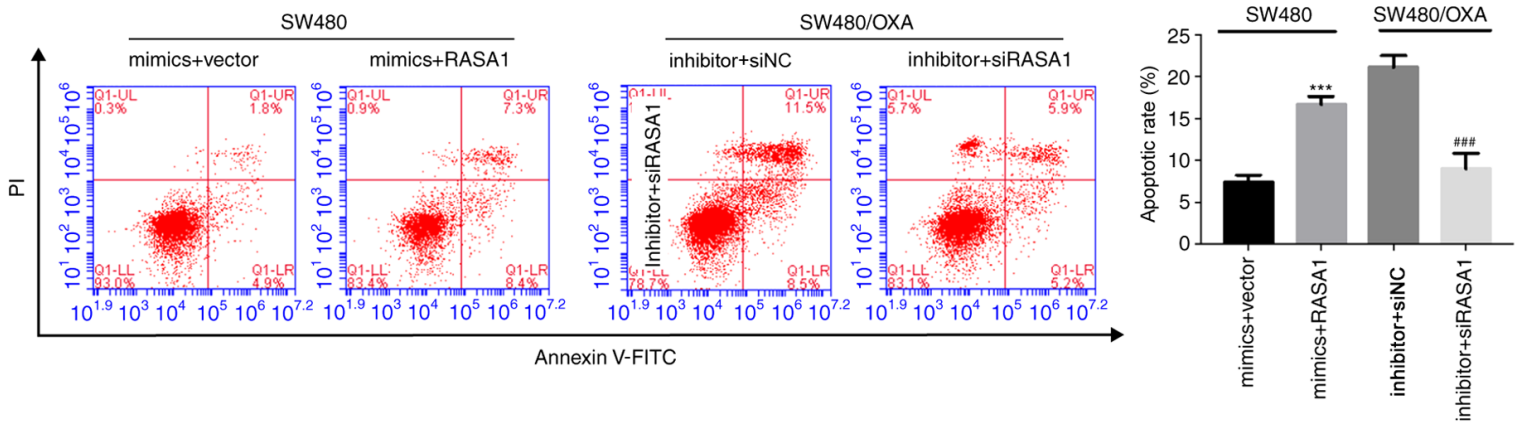

$\mathrm{D}$
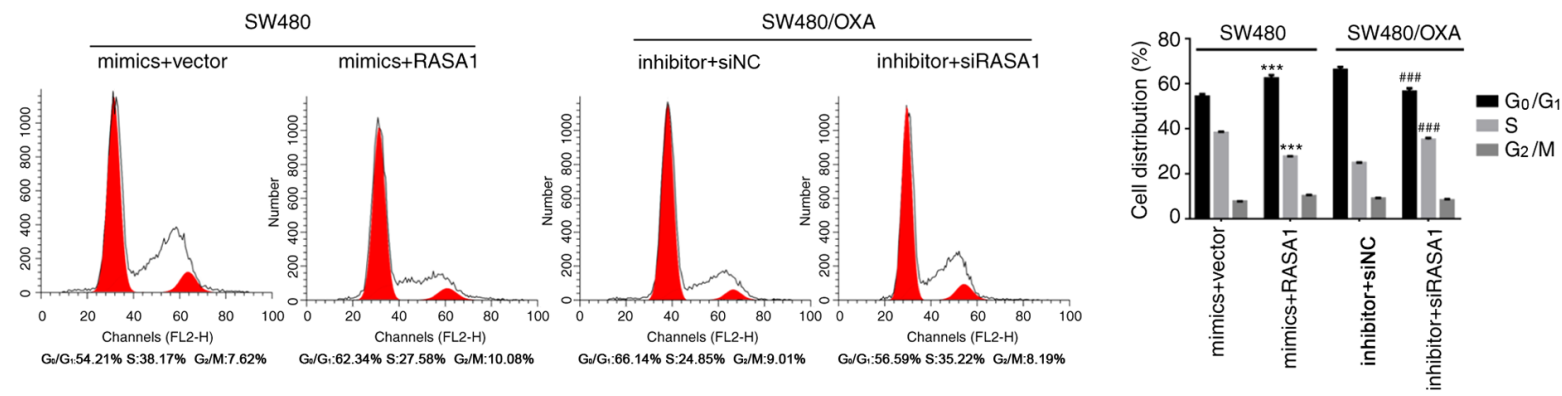

Figure 4. Downregulation of miR-188-5p promotes SW480/OXA cell apoptosis by increasing RASA1 expression. (A) RASA1, p21, and Ras-GTP protein expression in SW480 or SW480/OXA cells following co-transfection with RASA1 plasmid or RASA1 siRNA and with miR-188-5p or inhibitor. (B) Hoechst 33342 staining in the different transfection groups. Scale bar, $100 \mu \mathrm{m}$. (C) Apoptosis rate in the different transfection groups. (D) Cell cycle distribution was analyzed by flow cytometry. ${ }^{* * *} \mathrm{P}<0.001$ vs. mimics + vector group; ${ }^{\# \#} \mathrm{P}<0.001$ vs. inhibitor + siNC group. miR, microRNA; oxa, oxaliplatin; RASA1, Ras GTPase-activating protein 1; NC, negative control siNC, siRNA negative control; PI, propidium iodide; FITC, fluorescein isothiocyanate.

the protein expression levels of Ras-GTP compared with NC mimics; whereas miR-188-5p inhibitor significantly decreased the expression of Ras-GTP compared with NC inhibitor $(\mathrm{P}<0.001$ and $\mathrm{P}<0.01$, respectively; Figs. $2 \mathrm{~A}$ and $\mathrm{S} 1 \mathrm{~A})$.The expression of Ras-GTP changed following miR-188-5p mimic or inhibitor transfection (Figs. 2A and S2A).

Moreover, in the Hoechst 33342 staining and Annexin V/PI assays, transfection with miR-188-5p mimics inhibited cell apoptosis compared with transfection with NC mimics, whereas miR-188-5p inhibitor promoted cell apoptosis compared with $\mathrm{NC}$ inhibitor in SW480 and SW480/OXA cells $(\mathrm{P}<0.01$ and $\mathrm{P}<0.001$, respectively; Figs. $2 \mathrm{~B}$ and $\mathrm{C}$ and $\mathrm{S} 2 \mathrm{~A}$ ). These data suggested that targeting miR-188-5p promoted apoptosis in both SW480 and SW480/OXA cells.

The effect of miR-188-5p on cell cycle progression was also determined. Transfection with miR-188-5p mimics reduced the $G_{0} G_{1}$-phase cell population and increased the proportion of cells in the $S$ phase, while miR-188-5p inhibitor arrested cells at the $\mathrm{G}_{1}$ phase (Fig. 2D; all $\mathrm{P}<0.001$ ). These data suggested that targeting miR-188-5p delays the cell cycle.
S1B), compared with the respective negative controls. In addition, the overexpression of RASA1 decreased the expression of Ras-GTP, whereas siRASA1 transfection increased it, both in SW480 and SW480/OXA cells (Figs. 3B and S2B). and significantly Conversely, the expression of p21 decreased (Figs. 3B and S1B) following RASA1 silencing increased in RASA1-overexpressing SW480 and SW480/OXA cells $(\mathrm{P}<0.01$ and $\mathrm{P}<0.001$, respectively; Figs. $3 \mathrm{~B}$ and $\mathrm{S} 1 \mathrm{~B})$.

Furthermore, apoptosis was decreased following RASA1 silencing (Figs. 3C and D and S2B; P<0.005). However, RASA1 overexpression promoted apoptosis, both in SW480 and SW480/OXA cells (Figs. $3 \mathrm{C}$ and D and S2B; P<0.001). In addition, SW480 and SW480/OXA cells accumulated in the $\mathrm{G}_{0} \mathrm{G}_{1}$ phase of the cell cycle following RASA1 overexpression, and the frequency of S-phase cells was reduced (Fig. 3E; $P<0.001)$. By contrast, the frequency of $G_{0} G_{1}$ cells decreased, whereas the frequency of $\mathrm{S}$-phase cells increased following RASA1 silencing (Fig. 3E; $\mathrm{P}<0.001$ ). These findings further confirmed that RASA1 induced the cell apoptosis of colon cancer cells. 
Suppression of miR-188-5p promotes SW480/OXA cell apoptosis by increasing RASAl expression. Co-transfection experiments were performed to determine whether the increase in apoptosis induced by the miR-188-5p inhibitor was dependent on RASA1. As shown in Figs. 4A and S1C RASA1 and p21 were upregulated following $\mathrm{miR}-188-5 \mathrm{p}$ mimic transfection, while the transfection with miR-188-5p inhibitor inhibited the expression of RASA1 and p21, compared with cells transfected with the mimics and empty vector control $(\mathrm{P}<0.001)$.

Hoechst 33342 staining and Annexin V assays showed that overexpression of RASA1 enhanced apoptosis in miR-188-5p mimic-treated SW480 cells, compared with cells transfected with the mimics and empty vector control, whereas RASA1 silencing combined with miR-188-5p inhibitor transfection reduced the apoptosis rate (Figs. S2C, 4B and C; $\mathrm{P}<0.001$ ). Moreover, RASA1 overexpression increased the frequency of cells in the $\mathrm{G}_{0} \mathrm{G}_{1}$ phase while decreasing the frequency of cells in the $\mathrm{S}$ phase of the cell cycle in miR-188-5p mimic-transfected SW480 cells (Fig. 4D). By contrast, siRASA1 and miR-188-5p inhibitor transfection resulted in the opposite effect (Fig. 4D; $\mathrm{P}<0.001$ ). These results indicated that the inhibition of miR-188-5p induced SW480/OXA cell apoptosis by enhancing RASA1 expression.

\section{Discussion}

miR-188-5p was significantly upregulated in both SW480 and SW480/OXA cells. Transfection with miR-188-5p resulted in significant inhibition of SW480 and OXA-induced cell apoptosis, whereas both SW480 and SW480/OXA apoptosis significantly increased following miR-188-5p inhibition. Moreover, miR-188-5p transfection downregulated RASA1 expression. Moreover, the overexpression of miR-188-5p conferred OXA resistance, while downregulation of miR-188-5p sensitized resistant cells to OXA, which was blocked by inhibition of RASA1. In summary, the present study suggested that miR-188-5p could enhance colon cancer cell chemosensitivity by promoting the expression of RASA1.

Accumulating evidence has demonstrated the role of miR-188-5p in cancer development. However, the expression of miR-188-5p in OXA-resistant colon cancer cells has not been characterized. In the present study, the expression of miR-188-5p significantly increased in OXA-resistant colon cancer cells. A previous miRNA sequencing study by Zhao et al (14) involving 228 patients, six miRNA candidates, including miR-188-3p, were identified as strong predictors of patient survival. In addition, miR-188-3p promotes CRC cell migration both in vitro and in vivo partly by regulating MLLT4 expression, and high miR-188-3p expression is considered an independent prognostic factor of colon cancer (17). Moreover, a previous study reported the role of miR-188-5p in rectal cancer and response to neoadjuvant radio- and chemotherapy (22). The results of the present study were partly consistent with previous studies showing that miR-188-5p promoted colon cancer progression $(22,39,40)$. In the present study, miR-188-5p inhibition promoted apoptosis, whereas the upregulation of miR-188-5p inhibited apoptosis in SW480/OXA cells, suggesting that targeting miR-188-5p promoted apoptosis in both SW480 and
SW480/OXA cells. Moreover, cell cycle arrest in the $\mathrm{G}_{0} \mathrm{G}_{1}$ phase was induced, while apoptosis was increased following miR-188-5p inhibition. These results were consistent with previous studies showing that miR-188-5p controlled cell cycle progression via downregulation of multiple cyclins and cyclin-dependent kinase complexes involved in the $G_{1} / S$ transition $(20,41)$. Although previous studies have indicated that miR-188-5p serves as a tumor suppressor and is downregulated in multiple types of cancer $(15,42)$, miR-188-5p was upregulated in OXA-resistant colon cancer and RASA1 silencing abrogated the increase in cell apoptosis induced by the miR-188-5p inhibitor in the present study. Hence, miR-188-5p may enhance colon cancer cell chemosensitivity by promoting the expression of RASA1.

Of the several potential target genes for miR-188-5p predicted by TargetScan, PITA and miRDB Human database, RASA1 was selected for the present study. RASA1 acts as a suppressor of Ras function and enhances the weak intrinsic GTPase activity of Ras proteins, resulting in the inactive GDP-bound form of Ras (23). Previous studies have suggested that RASA1 acts as a tumor suppressor in hepatocellular carcinoma (42), human bronchial epithelial cells (43), breast cancer (44) and melanoma (45). In colon cancer cells, RASA1 is significantly downregulated, and has been identified as a target gene of miR-21, miR-223 and miR-335 $(34,36)$. Moreover, onco-miRNA molecules, such as microRNA-21 and microRNA-182 promote tumor angiogenesis or lymph node metastasis by targeting RASA1 $(37,38)$. p21 is a tumor suppresser gene that acts as negative regulator of the $G_{1} / S$ transition (46). Downregulation of p21 is observed in various human cancer types, including melanoma, gastric, ovarian and colon cancers (47). In the present study, the expression of miR-188-5p was negatively associated with that of RASA1 in OXA-resistant colon cancer cells. The overexpression of RASA1 promoted apoptosis, induced the expression of $\mathrm{p} 21$ and delayed cell cycle progression. Moreover, apoptosis in SW480 cells was reduced following miR-188-5p mimic transfection, while overexpression of RASA1 enhanced cell apoptosis in miR-188-5p mimic-transfected SW480 cells. By contrast, suppression of RASA1 abrogated cell apoptosis in miR-188-5p inhibitor-transfected SW480/OXA cells. These results suggested that miR-188-5p inhibition induced SW480/OXA cell apoptosis by enhancing RASA1 expression.

In conclusion, the present findings suggest that targeting miR-188-5p promoted apoptosis in SW480/OXA cells and suppression of miR-188-5p promoted SW480/OXA cell apoptosis by increasing RASA1 expression, thus providing new insight into treatment options for OXA-resistant colon cancer.

\section{Acknowledgements}

Not applicable.

\section{Funding}

This study was supported by The Guilin Scientific Research and Technology Development Plan Project (grant no. 20170109-50). 


\section{Availability of data and materials}

The datasets used and/or analyzed during the current study are available from the corresponding author on reasonable request.

\section{Authors' contributions}

HW conceived and designed the study, and drafted and revised the manuscript. XZ, XSL, ZS, SJ and XG performed the experiments. XKL, XX, and LZ analyzed data and prepared the figures. XZ and XSL edited and revised the manuscript. HW and XZ confirmed the authenticity of all the raw data. All authors read and approved the final manuscript.

\section{Ethics approval and consent to participate}

Not applicable.

\section{Patient consent for publication}

Not applicable.

\section{Competing interests}

The authors declare that they have no conflict of interest.

\section{References}

1. Siegel RL, Miller KD and Jemal A: Cancer statistics, 2020. CA Cancer J Clin 70: 7-30, 2020.

2. The Lancet Gastroenterology Hepatology: Colorectal cancer screening: Is earlier better? Lancet Gastroenterol Hepatol 3: 519, 2018.

3. Dekker E, Tanis PJ, Vleugels JLA, Kasi PM and Wallace MB: Colorectal cancer. Lancet 394: 1467-1480, 2019.

4. Lai M, Liu G, Li R, Bai H, Zhao J, Xiao P and Mei J: Hsa_circ_0079662 induces the resistance mechanism of the chemotherapy drug oxaliplatin through the TNF- $\alpha$ pathway in human colon cancer. J Cell Mol Med 24: 5021-5027, 2020.

5. Zhou Y, Zhang J, Wang K, Han W, Wang X, Gao M, Wang Z, Sun Y, Yan H, Zhang H, et al: Quercetin overcomes colon cancer cells resistance to chemotherapy by inhibiting solute carrier family 1, member 5 transporter. Eur J Pharmacol 881: 173185, 2020.

6. Meister $\mathrm{G}$ and Tuschl T: Mechanisms of gene silencing by double-stranded RNA. Nature 431: 343-349, 2004.

7. Garofalo M and Croce CM: MicroRNAs: Master regulators as potential therapeutics in cancer. Annu Rev Pharmacol Toxicol 51: 25-43, 2011.

8. Xie Y, Tobin LA, Camps J, Wangsa D, Yang J, Rao M, Witasp E, Awad KS, Yoo N, Ried T and Kwong KF: MicroRNA-24 regulates XIAP to reduce the apoptosis threshold in cancer cells. Oncogene 32: 2442-2451, 2013.

9. Yamanaka S, Campbell NR, An F, Kuo SC, Potter JJ, Mezey E, Maitra A and Selaru FM: Coordinated effects of microRNA-494 induce $\mathrm{G}_{2} / \mathrm{M}$ arrest in human cholangiocarcinoma. Cell Cycle 11: 2729-2738, 2012.

10. Zhu H, Wu H, Liu X, Evans BR, Medina DJ, Liu CG and Yang JM: Role of MicroRNA miR-27a and miR-451 in the regulation of MDR1/P-glycoprotein expression in human cancer cells. Biochem Pharmacol 76: 582-588, 2008.

11. D'Angelo E, Vicentini C, Agostini M, Kiss A, Baffa R, Scarpa A and Fassan M: MicroRNAs as tools and effectors for patient treatment in gastrointestinal carcinogenesis. Curr Drug Targets 16: 383-392, 2015.

12. D'Angelo E, Zanon C, Sensi F, Digito M, Rugge M, Fassan M, Scarpa M, Pucciarelli S, Nitti D and Agostini M: MiR-194 as predictive biomarker of responsiveness to neoadjuvant chemoradiotherapy in patients with locally advanced rectal adenocarcinoma. J Clin Pathol 71: 344-350, 2018.
13. Gao C, Zhou C, Zhuang J, Liu L, Liu C, Li H, Liu G, Wei J and Sun C: MicroRNA expression in cervical cancer: Novel diagnostic and prognostic biomarkers. J Cell Biochem 119: 7080-7090, 2018

14. Zhao L, Ni X, Zhao L, Zhang Y, Jin D, Yin W, Wang D and Zhang W: MiroRNA-188 Acts as tumor suppressor in Non-Small-Cell Lung cancer by Targeting MAP3K3. Mol Pharm 15: 1682-1689, 2018.

15. Peng Y, Shen X, Jiang H, Chen Z, Wu J, Zhu Y, Zhou Y and Li J: MiR-188-5p suppresses gastric cancer cell proliferation and invasion via Targeting ZFP91. Oncol Res 27: 65-71, 2018.

16. Li N, Shi H, Zhang L, Li X, Gao L, Zhang G, Shi Y and Guo S: MiR-188 inhibits glioma cell proliferation and cell cycle progression through targeting $\beta$-Catenin. Oncol Res 26: 785-794, 2018.

17. Pichler M, Stiegelbauer V, Vychytilova-Faltejskova P, Ivan C, Ling H, Winter E, Zhang X, Goblirsch M, Wulf-Goldenberg A, Ohtsuka M, et al: Genome-Wide miRNA Analysis Identifies miR-188-3p as a novel prognostic marker and molecular factor involved in colorectal carcinogenesis. Clin Cancer Res 23: 1323-1333, 2017.

18. Wang L and Liu H: MicroRNA-188 is downregulated in oral squamous cell carcinoma and inhibits proliferation and invasion by targeting SIX1. Tumour Biol 37: 4105-4113, 2016.

19. Fang F, Chang RM, Yu L, Lei X, Xiao S, Yang H and Yang LY: MicroRNA-188-5p suppresses tumor cell proliferation and metastasis by directly targeting FGF5 in hepatocellular carcinoma. J Hepatol 63: 874-885, 2015.

20. Zhang H, Qi S, Zhang T, Wang A, Liu R, Guo J, Wang Y and $\mathrm{Xu}$ Y: MiR-188-5p inhibits tumour growth and metastasis in prostate cancer by repressing LAPTM4B expression. Oncotarget 6: 6092-6104, 2015.

21. Jinlong S, Lin F, Yonghui L, Li Y and Weidong W: Identification of let-7a-2-3p or/and miR-188-5p as prognostic biomarkers in cytogenetically normal acute myeloid leukemia. PLoS One 10: e0118099, 2015.

22. Della Vittoria Scarpati G, Falcetta F, Carlomagno C, Ubezio P, Marchini S, De Stefano A, Singh VK, D'Incalci M, De Placido S and Pepe S: A specific miRNA signature correlates with complete pathological response to neoadjuvant chemoradiotherapy in locally advanced rectal cancer. Int J Radiat Oncol Biol Phys 83: 1113-1119, 2012.

23. Pamonsinlapatham P, Hadj-Slimane R, Lepelletier Y, Allain B, Toccafondi M, Garbay C and Raynaud F: p120-Ras GTPase activating protein (RasGAP): A multi-interacting protein in downstream signaling. Biochimie 91: 320-328, 2009.

24. Yang XY, Guan M, Vigil D, Der CJ, Lowy DR and Popescu NC: p120Ras-GAP binds the DLC1 Rho-GAP tumor suppressor protein and inhibits its RhoA GTPase and growth-suppressing activities. Oncogene 28: 1401-1409, 2009.

25. Calvisi DF, Ladu S, Conner EA, Seo D, Hsieh JT, Factor VM and Thorgeirsson SS: Inactivation of Ras GTPase-activating proteins promotes unrestrained activity of wild-type Ras in human liver cancer. J Hepatol 54: 311-319, 2011.

26. Mai A, Veltel S, Pellinen T, Padzik A, Coffey E, Marjomäki V and Ivaska J: Competitive binding of Rab21 and p120RasGAP to integrins regulates receptor traffic and migration. J Cell Biol 194: 291-306, 2011.

27. Sun D, Yu F, Ma Y, Zhao R, Chen X, Zhu J, Zhang CY, Chen J and Zhang J: MicroRNA-31 activates the RAS pathway and functions as an oncogenic MicroRNA in human colorectal cancer by repressing RAS p21 GTPase activating protein 1 (RASA1). J Biol Chem 288: 9508-9518, 2013.

28. Anand S, Majeti BK, Acevedo LM, Murphy EA, Mukthavaram R, Scheppke L, Huang M, Shields DJ, Lindquist JN, Lapinski PE, et al: MicroRNA-132-mediated loss of p120RasGAP activates the endothelium to facilitate pathological angiogenesis. Nat Med 16: 909-914, 2010

29. Organ SL, Hai J, Radulovich N, Marshall CB, Leung L, Sasazuki T, Shirasawa S, Zhu CQ, Navab R, Ikura M and Tsao MS: p120RasGAP is a mediator of rho pathway activation and tumorigenicity in the DLD1 colorectal cancer cell line. PLoS One 9: e86103, 2014.

30. Sharma SB, Lin CC, Farrugia MK, McLaughlin SL, Ellis EJ, Brundage KM, Salkeni MA and Ruppert JM: MicroRNAs 206 and 21 cooperate to promote RAS-extracellular signal-regulated kinase signaling by suppressing the translation of RASA1 and SPRED1. Mol Cell Biol 34: 4143-4164, 2014. 
31. Liu Y, Liu T, Sun Q, Niu M, Jiang Y and Pang D: Downregulation of Ras GTPaseactivating protein 1 is associated with poor survival of breast invasive ductal carcinoma patients. Oncol Rep 33: 119-124, 2015.

32. Li Y, Qiu C, Tu J, Geng B, Yang J, Jiang T and Cui Q: HMDD v2.0: A database for experimentally supported human microRNA and disease associations. Nucleic Acids Res 42: D1070-D1074, 2014.

33. Siderovski DP and Willard FS: The GAPs, GEFs, and GDIs of heterotrimeric G-protein alpha subunits. Int J Biol Sci 1: 51-66, 2005.

34. Gong B, Liu WW, Nie WJ, Li DF, Xie ZJ, Liu C, Liu YH, Mei P and Li ZJ: MiR-21/RASA1 axis affects malignancy of colon cancer cells via RAS pathways. World J Gastroenterol 21: 1488-1497, 2015.

35. Sun D, Wang C, Long S, Ma Y, Guo Y, Huang Z, Chen X, Zhang C, Chen J and Zhang J: C/EBP- $\beta$-activated microRNA-223 promotes tumour growth through targeting RASA1 in human colorectal cancer. Br J Cancer 112: 1491-1500, 2015.

36. Lu Y, Yang H, Yuan L, Liu G, Zhang C, Hong M, Liu Y, Zhou M, Chen F and Li X: Overexpression of miR-335 confers cell proliferation and tumour growth to colorectal carcinoma cells. Mol Cell Biochem 412: 235-245, 2016.

37. Zhang L, Zhan X, Yan D and Wang Z: Circulating MicroRNA-21 is involved in lymph node metastasis in cervical cancer by targeting RASA1. Int J Gynecol Cancer 26: 810-816, 2016.

38. Zhu YJ, Xu B and Xia W: Hsa-mir-182 downregulates RASA1 and suppresses lung squamous cell carcinoma cell proliferation. Clin Lab 60: 155-159, 2014

39. Yan S, Yue Y, Wang J, Li W, Sun M, Gu C and Zeng L: LINC00668 promotes tumorigenesis and progression through sponging miR-188-5p and regulating USP47 in colorectal cancer. Eur J Pharmacol 858: 172464, 2019.
40. Wu J, Chen Z, Liu W, Zhang Y, Feng W, Yuan Y, Ye J, Wang L, Cai S, He Y, et al: MicroRNA-188-5p targeting Forkhead Box L1 promotes colorectal cancer progression via activating Wnt/ $\beta$-catenin signaling. Oncol Res: Nov 23, 2020 (Epub ahead of print).

41. Wu J, Lv Q, He J, Zhang H, Mei X, Cui K, Huang N, Xie W, $\mathrm{Xu} \mathrm{N}$ and Zhang Y: MicroRNA-188 suppresses G1/S transition by targeting multiple cyclin/CDK complexes. Cell Commun Signal 12: 66, 2014.

42. Chen YL, Huang WC, Yao HL, Chen PM, Lin PY, Feng FY and Chu PY: Down-regulation of RASA1 is associated with poor prognosis in human hepatocellular carcinoma. Anticancer Res 37: 781-785, 2017.

43. Hayashi T, Desmeules P, Smith RS, Drilon A, Somwar R and Ladanyi M: RASA1 and NF1 are preferentially Co-mutated and define a distinct genetic subset of smoking-associated non-small cell lung carcinomas sensitive to MEK inhibition. Clin Cancer Res 24: 1436-1447, 2018.

44. Suarez-Cabrera C, Quintana RM, Bravo A, Casanova ML, Page A, Alameda JP, Paramio JM, Maroto A, Salamanca J, Dupuy AJ, et al: A Transposon-based analysis reveals RASA1 Is involved in triple-negative breast cancer. Cancer Res 77: 1357-1368, 2017.

45. Sung H, Kanchi KL, Wang X, Hill KS, Messina JL, Lee JH, Kim Y, Dees ND, Ding L, Teer JK, et al: Inactivation of RASA1 promotes melanoma tumorigenesis via R-Ras activation. Oncotarget 7: 23885-23896, 2016.

46. Pines J and Rieder CL: Re-staging mitosis: A contemporary view of mitotic progression. Nat Cell Biol 3: E3-E6, 2001.

47. Cai K and Dynlacht BD: Activity and nature of p21(WAF1) complexes during the cell cycle. Proc Natl Acad Sci USA 95: 12254-12259, 1998. 